\title{
EDITORIAL
}

\section{Ventilatory assistance in patients with Duchenne muscular dystrophy}

\author{
F. Lofaso*\#, D. Orlikowski* and J-C. Raphael*
}

D uchenne muscular dystrophy (DMD) leads to progressive muscle weakness, causing respiratory failure [1, 2]. Untreated patients die at $<25$ yrs of age, and the mean age of death in the 1960s was 15 yrs. The introduction of mechanical ventilation has improved survival compared with historical controls [1-3]. This finding has led to general agreement that noninvasive ventilation prolongs life in patients with DMD, although no controlled studies have been performed to confirm this hypothesis [4].

Noninvasive mechanical ventilation is initially offered at night to treat sleep-related breathing disorders and hypoventilation, which are common in patients with DMD [5,6]. Breathing during sleep should be evaluated by polysomnography with continuous $\mathrm{CO}_{2}$ monitoring [7], or by continuous $\mathrm{CO}_{2}$ monitoring alone if polysomnography is not available. Overnight pulse oximetry combined with an early-morning arterial blood gas measurement (to detect a base excess) may also be used to monitor nocturnal ventilation.

As the disease progresses, hypoventilation starts to occur during the daytime, and additional daytime ventilation is offered [4]. When noninvasive mechanical ventilation is inadequately effective, poorly tolerated or not feasible owing to severe upper airway dysfunction, invasive ventilation via a tracheostomy may be considered [4]. Preliminary results suggested that mechanical ventilation by tracheostomy was more effective than noninvasive mechanical ventilation in preventing death, although disability was more severe in the tracheostomised patients [3].

Noninvasive ventilation via a mouthpiece provides round-theclock ventilatory assistance to patients with little or no vital capacity, which may improve the effectiveness of ventilation and, with assisted coughing techniques, delay the need for tracheostomy [4]. In the current issue of the European Respiratory Journal, Toussaint et al. [8] report on outcomes seen with this technique over a mean period of $8.6 \mathrm{yrs}$. This is the first study of outcomes in patients with DMD receiving 24-h noninvasive mechanical ventilation. Of the 42 patients, 10 died before tracheostomy: five from heart failure with

*Intensive Care Unit and Dept of Physiology-Functional Testing, Garches Institute, and Centre for Technological Innovation, Raymond Poincaré Teaching Hospital, Assistance Publique-Hôpitaux de Paris, Garches, and "Inserm UMR 651, Créteil, France.

CORRESPONDENCE: F. Lofaso, Service de Physiologie-Explorations Fonctionnelles, Hôpital Raymond Poincaré, 92380 Garches, France. Fax: 33 147107943. E-mail: f.lofaso@rpc.ap-hopparis.fr low-output syndrome, two from sudden death and three from acute respiratory failure. Whether these patients would have survived with tracheostomy cannot be determined. Another unanswered question is whether tracheostomy would have induced other causes of death in these patients. A randomised study comparing 24-h noninvasive ventilation via a mouthpiece and ventilation via a tracheostomy could answer these questions but is unlikely to be conducted, as it would raise formidable practical problems. However, the study by TOUSSAINT et al. [8] provides valuable information.

First, although comparisons with other studies are difficult, the mean survival was $31 \mathrm{yrs}$, suggesting that this approach did prolong life compared to the experience at the Newcastle Muscle Centre (Newcastle upon Tyne, UK), where home ventilation has been used since 1989 [1].

Secondly, respiratory failure caused $30 \%$ (three out of 10) to $50 \%$ (five out of 10, including the sudden deaths) of the deaths. Therefore, although respiratory failure was not completely eliminated by $24-h$ noninvasive ventilation, heart failure became the leading cause of death $(50-70 \%$ of deaths). However, because invasive mechanical ventilation may be an important optimiser of cardiovascular function [9], tracheostomy might conceivably also treat heart failure.

Thirdly, figure 4 in the report by TOussainT et al. [8] clearly suggests that, contrary to those authors' comment, transcutaneous $\mathrm{CO}_{2}$ tension remained above $5.99 \mathrm{kPa}$ and $6.65 \mathrm{kPa}$ in eight and four patients, respectively, after initiation of 24-h mouthpiece noninvasive ventilation.

Fourthly, in their discussion, ToussainT et al. [8] clearly outline the advantages of mouth breathing and the disadvantages of tracheostomy in patients with DMD. However, tracheostomy may also have a number of advantages: ventilation is more secure and effective, and direct airway suctioning can be performed during periods of airway hypersecretion. By reducing the work of breathing [10], tracheostomy may also reduce the number of hours of ventilatory support needed per day. In addition, swallowing can be preserved, and speech can be obtained during both inspiration and expiration in invasively ventilated patients [11].

Daytime noninvasive ventilation via a mouthpiece should not be viewed as an alternative to tracheostomy. There is widespread agreement that noninvasive ventilation is preferable to invasive mechanical ventilation at the early stages of DMD [4]. The study by TousSAINT et al. [8] demonstrates that patients with DMD should try diurnal noninvasive ventilation via a 
mouthpiece when nocturnal noninvasive ventilation becomes inadequately effective. However, the observational data reported by TOUSSAINT et al. [8] also highlight the limitations of this technique. The current authors believe that ventilation via tracheostomy still has a place in the management of DMD, albeit a far smaller one than in the past.

Determining the best date for tracheostomy in patients with DMD remains a challenge. TOUSSAINT et al. [8] show that death by respiratory failure can occur despite 24-h ventilation, adding to the evidence that respiratory failure develops insidiously in DMD. Furthermore, the large proportion of deaths due to heart failure invites questions about the possible effectiveness of invasive ventilation compared with noninvasive ventilation in improving or preventing heart failure. Thus, it is necessary to determine whether tracheostomy should be offered prophylactically, reserved for patients who are completely unable to breathe independently and/or performed when the first signs of heart failure develop. Studies of the prognosis and quality of life of tracheostomised DMD patients would help to answer this question [3, 12].

In addition, the decision to perform tracheostomy should only be taken with the patient's informed consent and after careful discussion of the impact of social factors and living arrangements. It should also be noted that the access to the resources necessary to support patients living at home with tracheostomy ventilation varies widely throughout Europe; mouthpiece ventilation may be especially valuable in countries where these resources are scarce.

\section{REFERENCES}

1 Eagle M, Baudouin SV, Chandler C, Giddings DR, Bullock R, Bushby K. Survival in Duchenne muscular dystrophy: improvements in life expectancy since 1967 and the impact of home nocturnal ventilation. Neuromuscul Disord 2002; 12: 926-929.
2 Jeppesen J, Green A, Steffensen BF, Rahbek J. The Duchenne muscular dystrophy population in Denmark, 1977-2001: prevalence, incidence and survival in relation to the introduction of ventilator use. Neuromuscul Disord 2003; 13: 804-812.

3 Raphael J-C, Chevret S, Chastang CI, Bouvet F, French Multicentric Group. A prospective multicentre study of home mechanical ventilation in Duchenne de Boulogne muscular dystrophy. Eur Respir Rev 1992; 2: 312-316.

4 Finder JD, Birnkrant D, Carl J, et al. Respiratory care of the patient with Duchenne muscular dystrophy: ATS consensus statement. Am J Respir Crit Care Med 2004; 170: 456-465.

5 Barbe F, Quera-Salva MA, McCann C, et al. Sleep-related respiratory disturbances in patients with Duchenne muscular dystrophy. Eur Respir J 1994; 7: 1403-1408.

6 Smith PE, Edwards RH, Calverley PM. Ventilation and breathing pattern during sleep in Duchenne muscular dystrophy. Chest 1989; 96: 1346-1351.

7 Lofaso F, Quera-Salva MA. Polysomnography for the management of progressive neuromuscular disorders. Eur Respir J 2002; 19: 989-990.

8 Toussaint M, Steens M, Wasteels G, Soudon P. Diurnal ventilation via mouthpiece: survival in end-stage Duchenne patients. Eur Respir J 2006; 28: 549-555.

9 Lemaire F, Teboul JL, Cinotti L, et al. Acute left ventricular dysfunction during unsuccessful weaning from mechanical ventilation. Anesthesiology 1988; 69: 171-179.

10 Chadda K, Louis B, Benaissa L, et al. Physiological effects of decannulation in tracheostomized patients. Intensive Care Med 2002; 28: 1761-1767.

11 Prigent H, Samuel C, Louis B, et al. Comparative effects of two ventilatory modes on speech in tracheostomized patients with neuromuscular disease. Am J Respir Crit Care Med 2003; 167: 114-119.

12 Raphael JC, Dazord A, Jaillard P, et al. Assessment of quality of life for home ventilated patients with Duchenne muscular dystrophy. Rev Neurol (Paris) 2002; 158: 453-460. 